\title{
Use of Chromatography in Animal Ecology
}

\author{
Emma B. Casanave, M. Soledad Araujo and Gustavo H. López
}

Additional information is available at the end of the chapter

http://dx.doi.org/10.5772/48669

\section{Introduction}

There are several applications of chromatographic science in the field of animal ecology, biology and physiology. One of the most spread uses is identifying the bile acid profiles either by Thin Layer Chromatography, Gas chromatography or High Performance Liquid Chromatography in wild collected feces.

The analysis of wild collected feces is a fundamental ecological tool, applied in studies about population size, diet analysis and to identify the presence of a species in a certain area. Moreover, it is useful when it is necessary to monitor those species which are elusive, difficult to observe, threatened, nocturnal or sympatric, or exist in low densities. The identification can be done by external physical characteristics such as size, shape, odor and color, or through specific signals associated with the deposition of feces, for example tracks and scrapes [1,2]. However, this technique is sometimes useless because of the difficulties that exist in the correct identification of feces. Often, this sort of evidence is not present mainly because many of these external characteristics are sensitive to environmental conditions such as heat, desiccation or fast decomposition in humid and rainy regions, and can be affected by another type of factors: health, diet, size and age of the individual $[3,4]$.

Because of these reasons is that other techniques become necessary. The use of chromatographic techniques to identify or confirm the identity of wild collected feces is of great importance for biologists, because invasive procedures such as capture and manipulation are avoided. During the last years, the chromatographic determination of fecal bile acids has become a more precise method to identify unknown feces from the wild. The comparison of the whole pattern of fecal bile acids between field-collected scats and scats with known origin allows identifying the species. It has been demonstrated in several studies that fecal bile acids and their relative concentration follow patterns that are species-specific, particularly for mammals [5-7], including our recent studies in Xenarthra species [8]. 
In this chapter we will discuss the general chromatographic methods commonly used for the analysis of bile acids in biological samples. Moreover, we will emphasize the TLC and HPLC methodologies we used at our laboratory and the most relevant results we obtained, applied to the identification of mammal species, particularly of the Magnaorden Xenarthra (Mammalia).

\section{Bile acids}

Bile acids are the main components of bile and are among the first products isolated in a pure form, generally from gallbladder bile where it is present in high concentrations. They are acidic steroids produced during cholesterol metabolism in the liver and are secreted in the gallbladder or in the intestine.

Bile acids are produced by all vertebrates and show a great structural diversity among different species $[9,10]$. Indeed, no other class of small molecules shows such striking variety across vertebrates. The diversity in bile salt chemical structures originates from differences in the two basic structural components of bile salt molecules: the 19-carbon $\left(\mathrm{C}_{19}\right)$ steroid nucleus and a side-chain. In all bile salts characterized to date, the four-ring cyclopentanophenanthrene ('steroid') nucleus (rings labelled A, B, C and D) is fully saturated. The A/B ring juncture is cis in most bile salts but trans in some species, a shift that greatly influences the overall shape of the steroid nucleus. A/B trans $(5 \alpha)$ bile salts have an extended, planar orientation of the steroid rings, while $A / B$ cis $5 \beta$ ) bile salts have a 'bent' orientation of the A ring relative to the other three rings [10].

The structural variation in the $\mathrm{C}_{19}$ of the steroid nucleus would be stereochemistry of the $\mathrm{A} / \mathrm{B}$ ring juncture, sites of the oxo or hydroxy groups and orientation of hydroxyl groups $(\alpha$ or $\beta$ ). The structural variation in the side chain includes the length, the presence and orientation of $\mathrm{OH}$ groups, the presence of unsaturation, the stereochemistry of the $\mathrm{C}_{25}$ carbon atom and the site of carboxyl group in bile acids and $\mathrm{OH}$ group in bile alcohols [9, 10]. Other than length, further structural variation in the side-chain includes the presence and orientation of hydroxyl groups, the presence of unsaturation in the side-chain, and above all, the substituent on the terminal carbon atom, which are a hydroxyl group in bile alcohols and a carboxyl group in bile acids. Side-chain length and the state of oxidation at $\mathrm{C}_{27}$ is used to assign bile salts to three broad classes: $\mathrm{C}_{27}$ bile alcohols, $\mathrm{C}_{27}$ bile acids and $\mathrm{C}_{24}$ bile acids [10]. The structure of the side chain determines the class of compound; bile acids have a carboxyl group at the end of the chain and bile alcohols have a primary alcohol group [11] (Fig. 1).

Unlike the majority of biological small molecules, whose structures have remained constant since the formation of prokaryotic and eukaryotic cells, the molecular structure of bile acids show a distinctive evolution which parallels that of the vertebrate species which formed them (from $\mathrm{C}_{27}$ bile alcohols, $\mathrm{C}_{27}$ bile acids to $\mathrm{C}_{24}$ bile acids). The progressive nature of bile acid evolution is detectable between different genera, between members of different families and members of different orders [10]. 


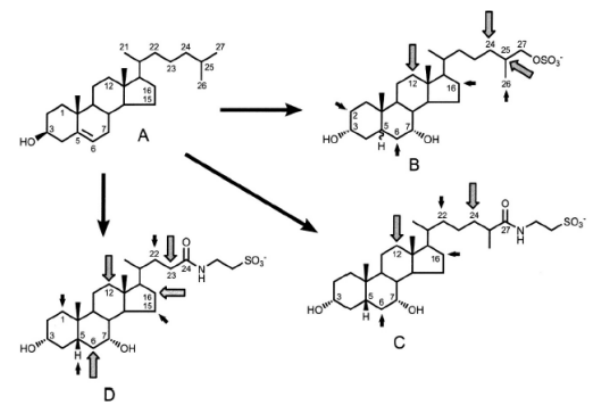

Figure 1. Sites of hydroxylation of the three main classes of bile salts. A: cholesterol; B: $\mathrm{C}_{27}$ bile alcohols (sulfates); C: $\mathrm{C}_{27}$ bile acids (as tauroamidated) and D: $\mathrm{C}_{24}$ bile acids (also as tauroamidated) (from [11]).

Conjugation of bile acids with the amino acids glycine and taurine occurs in the liver, before storage in the gallbladder and subsequent secretion into the duodenum via the bile duct. Within the intestinal lumen, bile acids interact with lipases and assist the lipolysis and absorption of fats, including fat-soluble vitamins, by the formation of mixed micelles. During enterohepatic circulation, the primary bile acids, CA and CDCA which are both synthesized in the liver may be modified by intestinal bacteria to form secondary bile acids, mainly DCA, LCA and UDCA (Fig. 2). In the colon of animals with a cecum, anaerobic bacteria remove the hydroxyl group at $C_{7}$ to form 7 -deoxy bile acids. In the side chain, bile acids suffer deconjugation [11, 12-14].

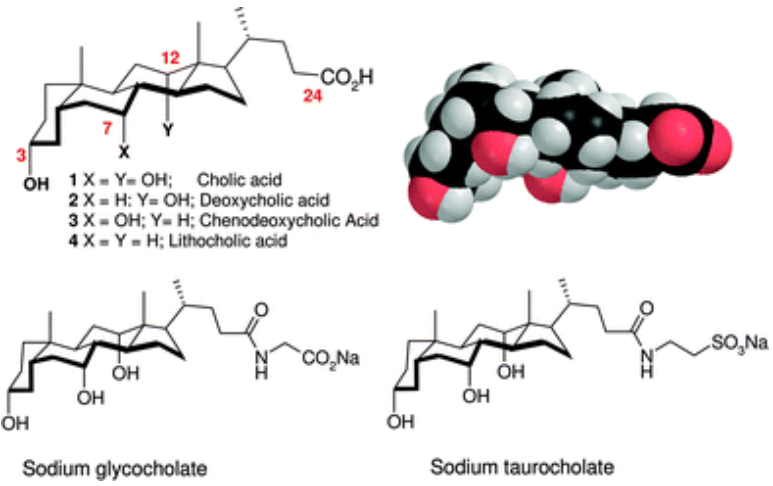

Figure 2. Structure of the main primary and secondary bile acids in mammals (from [15]).

Fecal bile acid patterns are complex due to bacterial metabolism during intestinal transit, which gives mono-, di- and/or -trioxo compounds, and also iso-( $3 \beta$-hydroxy), urso-(7 $\beta$ hydroxy) and lago-(12 $\beta$-hydroxy) bile acids [16-18]. The colon bacteria deconjugate bile acids. The majority of the unconjugated bile acids are $7 \alpha$-dehydroxylated, being LCA and DCA the predominant secondary fecal bile acids. They are reabsorbed from the colon, modified by hepatic enzymes and circulate in the enterohepatic circulation. Bile acids in the jejunum remain conjugated because of the absence of bacteria in the small intestine [19]. Moreover, in feces, certain bile acids are firmly bound to bacteria [12]. 


\section{Bile acid analysis}

The simultaneous separation and quantification of bile acids and their conjugates are challenging and have always presented technical difficulties due to marked differences in physicochemical properties, the presence of isomeric forms, their structural similarity or their relatively low concentrations in some biological samples [14, 20].

The most used techniques for the analysis of bile acid patterns in different biological samples include TLC coupled to densitometry, colorimetry, fluorimetry [5, 7, 21, 22], GC [16, 23, 24] and HPLC [25-27]. Moreover, non-chromatographic methods have been developed non chromatographic methods such as enzymatic, immunological and electrochemical ones. However, several of those methods are either not sensitive or are non-specific and have been displaced by GC, HPLC and radioimmunoassay. Radioimmunoassay, although it is highly sensitive, is not quite specific and overlaps of different bile acids occur [28]. On the other hand, both GC and HPLC are highly specific and sensitive to the extent of measuring even a few picomoles of bile acids [19].

Enzymatic methods using bile acid hydroxysteroid dehydrogenase are used for routine analysis of physiological fluids due to their simplicity. However, one of the main disadvantages is that differentiation of individual bile acids is not possible because total bile acids, rather than individual bile acids, are quantified [14].

Immunoassays of bile acids in different matrixes have provided relatively simple and sensitive methods for determining the concentration of selected sub-groups of bile acids. However, these assays do not provide full data for each of the individual bile acids.

Bile acids are haptens, nonantigenic low molecular weight, therefore complexes of bile acids covalently bound to large immunogenic molecules, for example bovine serum albumin, are required to obtain specific antibodies. In early experiments, $\left[{ }^{14} \mathrm{C}\right]-$ or $\left[{ }^{3} \mathrm{H}\right]$-labelled bile acids were used as tracers for analysis of serum bile acids, whereas [ $\left.{ }^{125} \mathrm{I}\right]$-labelled tracers were used in later publications. RIA can detect bile acids at the picomol level and are useful for high-throughput analysis. RIA for different bile acids in serum was described early for CA [29] and for CDCA, UDCA and LCA [30].

EIA use a hapten-enzyme conjugate as a tracer instead of radioisotope-labelled antigens. The construction of an effective EIA system requires the preparation of an enzyme-labelled antigen that possesses a high specific activity without decreasing the enzyme activity or immunoreativity. The labelling enzymes that have been employed for these assays include alkaline phosphatase and peroxidase [31]. Therefore, a quality antibody, preferably a monoclonal one, is very important for reliable analysis of bile acids in biological fluids.

The drawback of most RIAs and EIAs remains the limited specificity of the antibody. Usually, antibodies demonstrate high structural specificity for bile acids but often there is a low level of cross-reactivity with bile acids having similar structure to the target bile acid. 


\section{Sample collection}

The collection of samples for analysis is a critical step and constitutes a potential source of variation. For this reason, the procedure must be standardized. To determine the fecal bile acid pattern, it is necessary to collect feces from a known origin of the species under study. These reference feces can be collected in zoos, reserves or any place where one can assure the procedence of the fecal material. Moreover, feces can be collected during the capture and handling of a wild individual.

Feces are put in paper bags with silica gel so as to avoid humidity. They are correctly identified with: number, collector, date and place of collection, GPS coordinates, a microhabitat description, fecal external aspect (fresh, old, very old) and any information that it is considered relevant. Unknown feces from the study area, should match the external physical characteristics of the species under study, shape, size, colour and odour.

In our study, we analyzed the fecal bile acid patterns of different species from the Magnaorden Xenarthra (Mammalia). Our aim was to identify wild collected feces through their fecal bile acid patterns, and find in chromatography, a simple and rapid ecological tool.

For this purpose, samples were collected in zoos, reserves from the wild in different areas of Argentina. The studied species were: pichi (Zaedyus pichiy), screaming hairy armadillo or crying armadillo (Chaetophractus vellerosus), big hairy armadillo (Chaetophractus villosus), southern long-nosed armadillo (Dasypus hybridus), giant armadillo (Priodontes maximus), southern tamandua (Tamandua tetradactyla), giant anteater (Myrmecophaga tridactyla), southern three-banded armadillo (Tolypeutes matacus) and six-banded armadillo (Euphractus sexcinctus).

\section{Sample pre-treatment and solvent extraction}

The method of extraction will depend on the physicochemical properties of the biological material to be analyzed. Solid materials, such as feces, present particular problems due to the need of disrupting the matrix before extraction, either by homogenization, saponification, enzymatic digestion or lyophilization. Bile acids are hydrophobic steroids and hence, capable of extraction from aqueous media by organic solvents. The ease of extraction of bile acids from biological samples depends on four factors: the extent and type of conjugation, the number of hydroxyl groups, the state of ionization of the carboxyl group and the degree of binding to proteins. An increase in the number of hydroxyls or the presence of sulfates, glucuronides and to a lesser extent, glycine and taurine conjugates, will greatly increase the relative polarity (water solubility) of the bile acid. The state of ionization primarily depends upon the $\mathrm{pK}$ of the terminal group of the side chain, which varies from around 5.8 for the carboxyl group in the free bile acids to 4.3 for the glycine-conjugates and 1.9 for the sulfonic acid group of the taurine-conjugates. Although it would be expected that a decreased $\mathrm{pH}$ would favor extraction of bile acids because of the suppression of ionization, in fact bile acid extraction is, in many instances, favored at alkaline $\mathrm{pH}$, presumably because of the reduced protein binding that occurs [12]. 
Although there are a wide variety of methods available for the extraction of bile acids from biological materials, there is no general method that covers all eventualities. For that reason, the researcher must establish the solvent system which best fits to the aim of each particular study.

Previously to the extraction step, feces are dried in oven at $30^{\circ} \mathrm{C}$ for one day to eliminate humidity and are stored in hermetic flasks in a dry and dark place. Feces are crushed with the use of a mortar and pestle and sieved with a fine mesh. They are macroscopically observed and rests of preys (plants, seeds, insects, bones, feathers) are eliminated. Fecal extracts must be filtered and concentrated to an adequate final volume, preferably under nitrogen stream.

For the extraction step, the ratio solvent/sample should be kept high, 20:1 or 10:1 (v/w). In our study, we extracted 1 gram of the fecal powder with a mixture of benzene:methanol (1:1 $\mathrm{v} / \mathrm{v})$. With the use of this solvent system, we could extract from feces the highest number of compounds in a single step. In addition, we also tested another extraction solvent system composed of dichloromethane:methanol $(1: 1 \mathrm{v} / \mathrm{v})$. We replaced benzene with dichloromethane because it has the same extractive properties as benzene but is less toxic and thus easier to handle [3]. For HPLC, dried fecal extracts were resuspended in methanol and filtered with a polytetrafluorethylene filter of $0.45 \mu \mathrm{m}$ before injection.

\section{Thin-layer chromatography}

TLC of bile acids has been used to differentiate feces of several mammal species, mainly carnivores such as the lesser grisson (Galictis cuja), guiña cat (Leopardus guigna), red fox (Lycalopex culpaeus), grey fox (Lycalopex griseus), pampas's fox (Lycalopex gymnocercus), puma (Puma concolor), jaguar(Panthera onca), snow leopard(Panthera pardus ciscaucasica), pandas and different species of bears [6, 7, 32-37]. It had also been applied to a wide variety of other species: manatees [38], sperm whales [32], storks and herons [39]. Our study was the first one which reported the use of TLC to differentiate Xenarthra species, through their fecal bile acid patterns [8].

TLC is a type of planar chromatography in which the stationary phase is a solid adsorbent of fine particles, and the mobile phase is liquid. Aluminum or glass plates are used and they are covered with a fine and uniform layer of the stationary phase which is generally, silica. In some cases, it is chemically modified so as to provide suitable resolution conditions depending on the analyte. The correct choice of the mobile or stationary phase is essential to obtain an efficient separation of compounds to be analyzed [40-42]. Mobile phase generally consists of a solvent system composed of 2, 3 or 4 components which vary in their polarity and selectivity and they can include water, organic solvents and buffers [43, 44]. Silica gel is the most common adsorbent used in TLC as stationary phase, especially for the identification and separation of steroids such as bile acids in several biological samples, due to its adsorptive properties, great active surface and high pore size [45]. 
TLC separation mechanism consists of the differential migration of the mixture components through the chromatographic plate, dragged by the moving solvent; the distance travelled by each compound depends on its chemical structure and its affinity for both phases. Several mechanisms are involved in the separation process and predominant forces depend on the phases and solutes properties $[43,44]$.

Although various separation techniques are commonly applied for the determination of bile acids in biological samples, TLC offers practical advantages, mainly its simplicity, economical equipment needed, ease of operation, short analysis time and high efficiency in analysing simultaneously a large number of samples $[43,46]$. It enables reliable separation and analysis of a wide variety of compounds from different types of biological samples. Moreover, this technique is versatile because it can be modified using different types of mobile and stationary phases and visualizing methods [43, 45].

The choice of the correct stationary and mobile phases is of great importance and it depends upon the type of sample and the objectives of the study. Thus, the greatest problem in using TLC in specific cases, such as bile acid analysis, is the selection of suitable mobile phases [47].

\subsection{Experimental protocol}

Since there are a great variety of TLC methodologies to separate bile acids, in this section we report the technique used at our laboratory, which allows us to resolve the highest number of compounds in a single chromatographic run. The best results were obtained using HPTLC silicagel $60 \mathrm{~F}_{254}$ plates with aluminium base of $20 \times 20 \mathrm{~cm}$, with a bed thickness of 0.2 $\mathrm{mm}$. We follow the protocol cited in [5]. Each sample extract and standards for the most common bile acids for mammals are spotted on the plates with the use of a capillary tube: LCA, TCA, GCA, CA, CDCA, DCA, DHCA, GCDCA and CHOL. Bile acid standard stock solutions are prepared in methanol at a concentration of $0.1 \%$. We tested the use of different sample $(75,90,105,120,150$ and $180 \mu \mathrm{l})$ and standard $(7.5,15,22.5$ and $30 \mu \mathrm{l})$ quantities being spotted on the plates, so as to standardize the optimal concentrations for a better visualization.

As development solvent we use a mixture of toluene:aceticacid:water in a proportion of 5:5:1.5 v/v. We first saturate the mobile phase with water vapour and then we extract the rest of the liquid water. This gives more reproducibility to the analysis since variations in ambient humidity and temperature can alter the results.

Bile acid spots are visualized by spraying the plates with a revealing solution of anysaldehide:glacial acetic acid:sulphuric acid in a concentration of 0.5:50:1 v/v; plates are heated in oven at $150{ }^{\circ} \mathrm{C}$ for 15 minutes.

The bile acid pattern of each species is determined by the comparison of $\mathrm{R}_{\mathrm{f}}$ values (relation between distance travelled by the compound and distance travelled by the eluent) and colour of the compounds with those of standard solutions. The relative intensity (concentration) of sample spots helps in the identification of the fecal bile acid pattern of each species. 


\subsection{Results and discussion}

In our study, we could differentiate all the Xenarthra species through their fecal bile acid patterns. We detected 15 compounds in feces; seven bile acids, seven unidentified compounds and cholesterol.

Moreover, we showed the resolution of TLC because some pairs of bile acids with very similar $R_{f}$ values, CDCA-DCA and CA-GCA, were discriminated. Although the $R_{f}$ value is the main parameter to identify a compound, several other characteristics of the bands had to be carefully analysed in the chromatographic plates to determine bile acid patterns.

Some standard $R_{\mathrm{f}}$ values partially overlapped; however, we established a $\mathrm{R}_{\mathrm{f}}$ range for each compound and, together with their colour, the spots were correctly identified. The concentration of the compounds was proportional to the relative intensity of each band in the chromatographic plate, as determined visually. Specific colours obtained through the oxidation by anisaldehyde for each bile acid allowed the identification of the spots in the plates. DHCA differed from the other standards because it showed two and, in some cases, three bands with a distinctively orange colour. Standards which showed more intense colours were LCA (dark green), TCA (blue-grey) and CHOL (dark pink); the rest of the bile acids had less intense colours (Fig. 3).

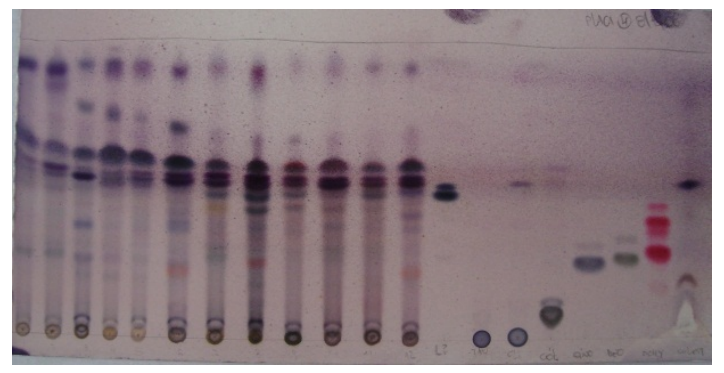

Figure 3. A chromatographic plate revealed with anisaldehyde:glacial acetic acid:sulphuric acid (0.5:50:1 v/v), shows bands of different colours. Left: 12 sample lines. Right: 8 standard bile acids.

Therefore, we stress the importance of spotting standard solutions together with the samples in each chromatographic plate to correctly identify compounds.

Even if TLC of fecal bile acids offers practical advantages such as simplicity and ease of operation $[43,46]$, like any laboratory technique it needs much of practical knowledge and skills [7] and it requires a careful and detailed analysis from the researcher.

In our study, TLC was useful to identify feces from Xenarthra species as it allowed the extraction, visualization and identification of fecal bile acids from individuals of all the studied species.

For several years, TLC has been used to identify wild-collected feces, mainly for species with low contents of vegetal material in their diets, such as carnivores [5, 35, 48, 49]. Nevertheless, in [34] they were not able to differentiate feces from Lycalopex culpaeus and 
L. griseus in Chile; they argued that there was too much variability in the spot pattern even among feces from the same individual. In spite of that, in [3] they could discriminate feces from both species. Moreover, in [6] authors demonstrated that bile acid patterns were specific for some threatened carnivore species in Chile.

Several factors should be considered when analysing TLC results. One of them is the concentration of the sample; in the case of our study, samples from captive animals showed more intense bands while samples from wild animals had little concentration of some bile acids, making the correct identification sometimes difficult. However, we could find the optimal concentration, i.e., the concentration that allowed the detection of the compounds in the chromatographic plates, for wild and captive animals, being higher for wild ones.

A second factor that should be considered, especially when working with wild animals, is the effect of the type of diet, which has been reported in other investigations. It is possible that the presence of chemical substances in the feces, as products of the diet, has an effect on the detectability of bile acids, masking the spots [34] as it was observed previously, for example in coyotes [50].

Xenarthra species are omnivores and carnivores-omnivores [51], including invertebrates, small vertebrates, carrion, plant roots, tubers and seeds in their diets [52-55]. During plate running, other colour bands were observed; they corresponded to plant pigments which were yellow or orange for captive animals and green for wild ones. Differences in extract colours among species may reflect variations in the diet composition, mainly due to vegetal pigments which are naturally colored contrary to bile acid which are uncolored. Although we found some plant material in the feces, they had only small amounts and there was no evidence that prey items interfere with bile acid identification.

Age of wild-collected scats and thus, weathering, plays an important role in the analysis of feces by TLC $[7,36]$. Some authors $[7,35,48,56]$ suggested that feces weathering can lower the concentration of bile acids in scats, leading to their erroneous identification. In our study, it was demonstrated that bile acids were clearly identified even in two-year old feces.

Further, small sample size can worsen TLC performance [36, 56, 57]; however, this was not the case of our study since we used sufficient quantity of dry pulverized fecal material to allow a good detection of the compounds [5].

The experience of the analyst in applying TLC is also important. For example, the spraying with the visualizing agent is not always uniform and some areas of the chromatographic plate may be uncoloured, resulting in an incorrect interpretation of the bands.

Several authors reported different TLC methods for the analysis and separation of bile acids. In [58] they reported a solvent system which allows group separation; free bile acids are separated with the first solvent system acetic acid:carbón tetrachloride:di-isopropyl ether:iso-amyl acetate:n-propanol:benzene (5:20:30:40:10:10 v/v), then with the second solvent system propionic acid:iso-amyl acetate:water:n-propano (15:20:5:10 v/v) they 
separated taurine and glycineconjugated standard bile acids. In [21], authors reported 15 solvent systems to separate bile acids, being the most efficient the acidic one.

In [59] they reported two solvent systems. The first one (isopropanol:glacial acetic acid, 93:7 $\mathrm{v} / \mathrm{v}$ ) allows separation of free bile acids, glycine conjugates and taurine conjugates from one another, and a second solvent system consisting of hexane:methylethylketone:glacial acetic acid, 56:36:8 v/v (Petcoff's solution), for the separation of the four free bile acids found in human bile. Authors in [48] used a TLC methodology to identify the fecal bile acid patterns in several carnivore species; plates were developed in a paper-lined, equilibrated bath containing Petcoff's solution. After air drying, plates were visualized by spraying with a solution of acetic acid:sulphuric acid:anisaldehyde 50:1:0.5 (v/v) as revealing agent, and placing in a $120^{\circ} \mathrm{C}$ oven [60].

On the other hand, as cited in [33], they developed plates in Petcoff's solution and then visualized with a 50:1:0.5 v/v solution of acetic acid:sulfuric acid:p-anisaldehyde to identify bile acids from grizzly and black bear feces. In [35] they used used a developing bath of Petcoff's solution to run the plates and they visualized bile acid bands by spraying the plates with $50 \% \mathrm{v} / \mathrm{v}$ sulfuric acid, which allowed them to observe spot colors in cougar and jaguar feces [3]. Sulfuric acid was also present in our visualizing agent. [61] reported a method to separate standard bile acids consisting of a reversed thin-layer chromatography using a mixture of metanol:water as the solvent system, in the concentration range $50-75 \%(\mathrm{v} / \mathrm{v})$.

In [6] they reported a TLC method to determine the fecal bile acid patterns in carnivore feces. It consisted of an alkaline extraction to purify the bile acids from other lipophilic steroidal compounds; plates were eluted with Petcoff's solution and developed with a mixture of glacial acetic acid, water, sulfuric acid and 3,4-dimethoxybenzaldehyde.

From our results, and because TLC of fecal bile acids has proved to offer robust data to establish habitat use and to study food habits of some sympatric mammal species [6, 36], we assume that this technique would also be useful for future ecological studies in Xenarthra and in other species. Considering the scarcity of available information about some ecological and biological aspects of Xenarthra, these results, the first ones on the application of TLC for the identification of their feces, could be very important for future studies about the conservation, distribution and eco-physiology of this group.

\section{Gas chromatography}

GC and GC-MS have been widely used for quantitative and qualitative analysis of bile acids in different biological samples [10, 14, 37, 62-65]. In broad terms, GC works on the same principle as HPLC, however, in GC compounds are in gaseous form and an inert gas, like nitrogen or helium, is used for elution. The various compounds are resolved on the basis of their retention/elution behavior on the stationary phase of the column. There are two basic requirements for GC analysis, an appropriate column and derivatization of the compounds, to cause vaporization under the chromatographic conditions used [19]. 
Then, those techniques are limited by complex sample preparation, derivatization and most importantly, because requires the hydrolysis of conjugated bile acids into their free forms, prior to their analysis [66]; although GC-MS provides high sensitivity and resolution of isomeric bile acids, it is necessary to isolate and purify bile acids before analysis $[14,19]$.

Bile acids are present in unconjugated and/or conjugated form in biological fluids. They range from 1-2 $\mu \mathrm{g} / \mathrm{ml}$ in plasma and urine to significant amounts in the intestinal content and as much as $10 \mathrm{mg} / \mathrm{ml}$ in the gallbladder bile. Bile acids are present in association with proteins, sterols and their esters, free or esterified fatty acids, bile pigments and watersoluble small molecules, which must be either removed before chromatography or the chromatographic conditions should be such that these compounds do not interfere in bile acid analysis. Since bile acids are present mainly as glycine or taurine conjugate in plasma and bile, unconjugated in feces and unconjugated as well as conjugated with glycine, taurine, glucuronic acid and sulfuric acid in the urine, the methods for isolation of bile acids from these sources need to be appropriately modified [19].

However, a major difficulty in quantitative analysis of fecal bile acids is their strong binding with the bacterial debris in the stool, and quantitative extraction is difficult. Stool contains bile acids, neutral sterols, cholesterol and its bacterial metabolites, plant sterols and their bacterial metabolites and fatty acids, which need to be removed before GC. Since fatty acids are less strongly retained on the capillary columns, bile acids can usually be quantitated in their presence.

For GC fecal bile acid analysis, several methods have been reported and most of them are quite complex [23, 62,67].

In [68] they used continuous soxhlet extraction of aliquots of stool using chloroform and methanol. Extracts were derivatized and the methyl esters obtained were subjected to preparative TLC, respective bands were eluted and prepared for GC. On the other hand, in [23] they did a similar procedure but with tedious and longer steps. In alternate methods, bile acids from feces have been extracted with ammoniacal alcohol, methanol-hydrochloric acid, acetic acid-toluene, after removal of neutral sterols [23, 67].

In [19] they digested fecal samples with internal standard, extracted neutral sterols by repeated extractions, extracted bile acids with ethyl acetate, remove mineral acid and the residue was subjected to methyl ester formation; part of the aliquot was used for GC after trimethylsilylation. However, this method was not applicable to samples which contained conjugated bile acids.

Since the basic requirement for GC is that the compounds are in gaseous form at the column temperature, it is necessary to derivatize the polar functional groups. The carboxyl group in bile acids is often converted into the methyl ester. In addition, other bile acid esters have been used for GC, such as ethyl, n-propyl, isobutyl and n-butyl esters. Moreover, other derivatizations are common: of the hydroxyl group or the oxo group. 


\section{High performance liquid chromatography}

The first report of the application of HPLC for bile acid separation was in 1976 [68 in 12]; and although its usefulness has been demonstrated for bile acid analysis in a wide variety of biological samples, up to the present it has never been used for the identification of species through their fecal bile acid pattern.

During the last years, HPLC has been one of the most used techniques for the analysis and identification of different lipid classes [44] and, particularly, for the separation and quantification of bile acids and their derivatives in different biological materials, due to its possibility to be coupled to a great variety of detectors [14, 27, 69-74]. HPLC presents several advantages in relation to other techniques such as high resolution, high sensitivity and specificity $[44,75]$.

A chromatographic system consists of four main components: a device for sample introduction, a mobile phase, a stationary phase (column) and a detector. The injector is simply required to allow introduction of the analytes into a flowing liquid stream without introducing any discrimination effects, being manually or automatically operated. The two components which are associated with the separation that occurs in a chromatographic system are the mobile and stationary phases. In HPLC the mobile phase is a liquid delivered under high pressure to ensure a constant flow rate, and reproducible retention times. The stationary phase is packed into a cylindrical column with a particulate support to which is bonded the stationary phase and it is capable of withstanding the high pressures which are necessary.

Detectors and columns vary depending on the objectives of the study and the type of sample. A small quantity of liquid sample is injected into the mobile phase which is flowing through the column, so the individual components of the mixture are separated [76, 77].

To take advantage of an HPLC column, it is necessary to use integrated chromatographic systems; all the components are specifically designed and coupled to get a maximum efficiency.

A chromatographic separation occurs if the components of a mixture interact to different extents with the mobile and/or stationary phases (Fig. 3), and therefore take different times to move from the position of sample introduction to the position at which they are detected. So, there are two extremes, as follows: a) all analytes have total affinity for the mobile phase and do not interact with the stationary phase; all analytes move at the same rate as the mobile phase, they reach the detector very quickly and are not separated, and b) all analytes have total affinity for the stationary phase and do not interact with the mobile phase; all analytes are retained on the column and do not reach the detector. The role of the analyst is therefore, based on a knowledge of the sample, to manipulate the properties of the stationary and/or mobile phases to move between these extremes and reach the desired separation [77]. 
Reversed-phase HPLC is the most used type of liquid chromatography for compound separation; it has been reported that almost $75 \%$ of all HPLC separations are done in the reverse mode. It is one of the most reliable and powerful methods for a direct and no destructive analysis of those compounds which are less volatile than others, polar and particularly, steroids and their conjugates [27]. Particularly, the resolution of compounds with similar polarities to bile acids results efficient if reversed-phase HPLC is used [78, 79].

The main characteristic of reversed-phase HPLC is that the mobile phase is more polar than the stationary phase, and therefore the components of the sample elute from the column in order of their hydrophobicity, number, position and configuration of hydroxyl groups in the steroid nucleus $[44,46,80]$.

The retention behavior of a certain compound in reversed-phase HPLC depends on its solubility in and the partition between mobile and stationary phases. The polarity of a molecule controls the solubility in the mobile phase while the hydrophobic surface plays a key role in the interaction with the stationary phase. Bile acids have a hydrophobic region in the $\beta$ face of the steroid nucleus, an area which is constant among common bile acids. Therefore, when increasing the number of $\mathrm{OH}$ groups in the $\alpha$ face of the molecule, increases the polarity and improve the solubility in an aqueous mobile phase. On the other hand, the presence of a $\beta \mathrm{OH}$ or carbonyl group, reduces the hydrophobic area of $\beta$ face, resulting in a diminished of retention time [79]. The most polar bile acids are trihydroxilated tauroconjugated ones and the least polar are free monohydroxylated bile acids [74, 79].

The choice of the mobile phase will depend not only on the characteristics of the analyte, but also on the detection mode. Almost all methods use an elution gradient with a binary or tertiary solvent system, which are combined depending on their polarities; so, a satisfactory resolution of different lipid classes is achieved [44].

\subsection{HPLC detectors}

In different biological samples, bile acids are present as a complex mixture with a wide variety of polarities due to the presence of conjugated and free forms. For that reason is that the choice of the detection system will be largely governed by the structures of the analytes $[14,27]$.

There are several detectors that can be coupled to an HPLC system. However, at the present it is not available a simple, sensitive and direct method for the simultaneous analysis and quantification of individual bile acids in different tissues and fluids [20].

UV detectors have been widely used for the determination of bile acids in biological samples $[25,38,81-83]$, mainly due to their relative low costs [44] and also because it allows the direct analysis of conjugated bile acids without prior derivatization [27, 71] and the reduction of the analysis time. However, HPLC-UV does not provide the required sensitivity and selectivity to detect the trace amounts of some bile acids or bile acid-sulfates in biological matrices [14, 20, 66, 73, 84], for example free bile acids [27, 80]. The simultaneous analysis of 
individual bile acids from a mixture presents several technical difficulties due to their similar and complex chemical structures, to their low UV absorbance, their low volatility, the presence of isomeric forms and the low concentration in certain type of samples [20, 27], particularly feces $[46,73,84]$.

In several studies, it is necessary to pretreat samples which involve extraction, purification and derivatization so as to increase sensitivity and specificity [40].

Moreover, HPLC has been coupled to other types of detectors, for example RID, FLD and Electrochemical Detector.

The use of RID is limited by poor sensitivity and unstableness of base-line in gradient elution conditions, which is necessary for the separation of a complicated mixture with relatively short analysis time. Furthermore, the use of FLD or electrochemical detection does not detect non-chromophoric bile acids without a suitable pre-column derivatization [27]. Methods for sample derivatization are complex, with fluorescing chemicals [71, 85] or other complex derivatizations [27], which can introduce contaminants and produce secondary incomplete reactions, involving more complexity and longer analysis times [14, 27, 44, 73]. That is why derivatization methods are not the choice for a comprehensive analysis of fecal bile acid patterns [84].

The majority of lipids show a maximum absorbance in the range of wavelengths from 190 to $210 \mathrm{~nm}$ [44]; particularly, bile acids show different capabilities of absorption to UV light depending on their structure. UV detection can be used at 200-210 nm with moderate sensitivity for glycine- and taurine-amidated bile acids, which can be analyzed directly without previous derivatization steps, using conventional UV detectors [27, 71]. Unconjugated bile acids and their sulfated and glycosylated conjugates have a markedly low absorbance in this range $[27,80]$.

For that reason, in certain cases, depending on the aim of the study, is necessary to pretreat samples. This involves steps of extraction, purification and derivatization to increase sensitivity and specificity [40]. Although a great variety of pre-column derivatizations have been used to increase detection of bile acids to UV light, the complete conversion of compounds it is not assured and in general, required long steps that finally led to the loss of sample [14, 27].

In recent years, another detector used is ELSD. Theoretically, this detector responds to all solutes that are not volatile and the response is proportional to the mass of the solute present. The eluent is atomized in a stream of nitrogen and the finely divided spray passes down a heated chamber during which time the solvent is evaporated. The removal of the solvent produces a stream of particles which pass through a collimated beam of light. The scattered light at an angle in relation to the incident light, is focused onto a photomultiplier tube and the output is processed in an appropriate manner electronically. For a linear response, the droplet size must be carefully controlled. The device is fairly compact and relatively simple to operate. Detector sensitivity is in the range of 10-20 ng of solute. However, the magnitude of the response varies widely between different substances. 


\subsection{Liquid chromatography-mass spectrometry}

Methods using FAB-MS and ESI-MS are applied in bile acid analysis. FAB-MS, however, has inferior quantitative capabilities and yields less intact ions (more in-source fragmentation) compared to ESI. Therefore, ESI-LC-MS remains a powerful technique for direct quantitative analysis of bile acids in biological matrices. Several methods have been developed and used to quantify bile acids in biological matrices using ESI-LC-MS [73, 86, 87].

The persistent need for rapid and sensitive methods has motivated efforts to exploit the high sensitivity, specificity and the minimal sample preparation requirements of HPLC-MS/MS for bile acid analysis in biological fluids, including the complex profile of secondary bile acids in feces resulting from colonic bacterial metabolism [88].

In conventional ESI the sample eluting from HPLC is pumped through a thin capillary (internal diameter approximately $0.1 \mathrm{~mm}$ ) which is raised to a high potential $(4 \mathrm{kV})$. Small charged droplets are sprayed from the ESI capillary into a stream of inert gas, generally nitrogen, at atmospheric pressure and travel down towards an orifice in the massspectrometer high-vacuum system. As the droplets traverse this path they become desolvated and reduced in size to such an extent that surface-coulombic forces overcome surface-tension forces and the droplets break up into smaller droplets. This process continues until they reach a point in which either an ion desorbs from a droplet or solvent is completely removed. This mechanism results in a beam of ions, which are directed to the mass spectrometer.

In a quadrupole mass analyzer MS/MS instrument, a precursor ion is mass-selected by first mass analyzer and focused into a collision cell preceding a second mass analyzer. The mass analyzers are arranged in series. Inert gas is generally introduced into the collision region and collisions occur between the precursor ion and inert gas molecules. In these collisions part of the precursor ions translational energy can be converted into internal energy, and as a result of single or multiple collisions an unstable excited precursor ions decompose to product ions. Product ions are mass-analyzed by the second mass analyzer [89].

MS/MS of steroids and bile acids has been an area of considerable study [90,91]. When analyzed by negative-ion FAB or ESI-MS, bile acids, steroid sulphates and steroid glucuronides give abundant $[\mathrm{M}-\mathrm{H}]-$ ions and few fragment ions. To gain structural information, MS/MS spectra are recorded. Any modification of the steroid skeleton or side chain would result in a change in the pattern of fragment ions [91].

LC-MS/MS is a simple, sensitive and rapid technique for the analysis of bile acids in complex matrix as feces [46].

\subsection{Experimental protocol}

In this section, we will describe the HPLC methods used to identify fecal bile acids in Xenarthra species. 
We used a HPLC Thermo Finnigan made up of a gradient quaternary pump, on-line degassifier, a thermostatic module for the column and an UV-Vis detector with double wavelength, set at 200 and $210 \mathrm{~nm}$. Analyses were performed on a reversed-phase C-18 column and a similar pre-column. We adapted the HPLC experimental protocol from [25] to our objectives.

Standard bile acids were CA, DCA, DHCA, CDCA, LCA, UDCA, GCA, sodium glicocholate, GDCA, GCDCA, TCA, TDCA, TLCA and CME, and CHOL. All were prepared in methanol at a concentration of $0.1 \%$; and, when it was necessary, at $0.2 \%$ and $0.4 \%$, and filtered through a $0.45 \mu \mathrm{m}$ syringe driven HPLC filter.

As mobile phase we used a solution of $0.3 \%$ ammonium carbonate in water/acetonitrile. The analyses were performed under a linear gradient constituted as follows: 73:27 v/v for 10 minutes, $68: 32 \mathrm{v} / \mathrm{v}$ for 10 minutes, $50: 50 \mathrm{v} / \mathrm{v}$ for 10 minutes and from 50 to $100 \%$ acetonitrile until 60 minutes. The flow rate was $0.8 \mathrm{ml} / \mathrm{min}$. Column temperature was set at $25-27^{\circ} \mathrm{C}$, and the sample volume injected was $20 \mu \mathrm{l}$. For the most retained compounds, solvent gradient was modified, gradually increasing from the minute 30 to the minute 60 from $50 \%$ to $100 \%$ acetonitrile.

We run a mixture of all standards before each series of samples, so as to compensate any possible variation in retention times due to column efficiency loss or environmental conditions. Sample peaks were tentatively assigned comparing their retention times with those of standard solutions, previously injected in the same conditions.

For purposes of quantification, calibration curves for all standards in methanol were constructed. Regression equations were obtained through linear regression analysis and applied to the peak area of each bile acid as a function of concentration.

In each chromatogram we measured the retention time $\left(R_{t}\right)$ expressed in minutes which is defined as the time that each compound spends in eluting from the column, the peak area (A) expressed in absorbance units (mAU), which determines the quantity of each compound present in the sample and the peak width measured at the base line $(\mathrm{W})$.

\subsection{Results and discussion}

HPLC has been applied in a great variety of studies with different aims; however it has never been used as an ecological tool for differentiating species through fecal bile acids. In our work, we could identify the fecal bile acid pattern for all Xenarthra studied species, allowing the differentiation of wild-collected feces. No differences were observed between males and females, or between captive and wild individuals of the same species. All the species presented DHCA, TCA, TLCA and LCA, besides from CHOL, and three unidentified peaks.

When we calculated the percentage composition of each bile acid in samples from different individuals of the same species, we could observe that the compounds were the same; 
however, their quantities vary between them. This can be due to intra and/or interindividual variations.

Variations in the composition of bile acids were reported for other biological samples. In [71] authors found differences in the hepatic composition of bile acids among different individuals of rats. Moreover, in [92] they recently reported diurnal variations, between morning and afternoon, in liver and plasma bile acids in laboratory mice. Bile acid circadian rhythm has been recognized for several years by various authors in different biological materials such as plasma, liver, gallbladder and intestinal contents. Our results coincide with those studies in which they did not report variations in the bile acid pattern of the species but only in the quantity of some of them [92].

Individual retention times for all 15 standard bile acids and CHOL, were obtained. A typical chromatogram of the mixture of standards shown in Fig. 4. Free bile acids, taurineconjugated and glycine-conjugated bile acids were resolved in less than 32 minutes, achieving also the resolution of DCA and GDCA acids which could not be separated in previous works $[25,80]$.

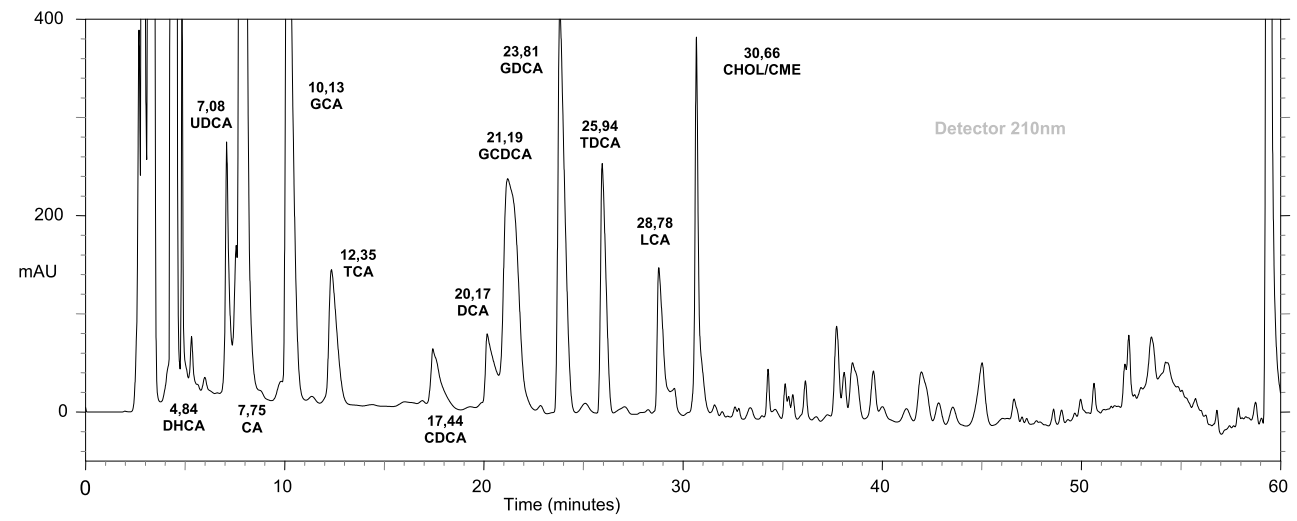

Figure 4. A typical HPLC chromatogram of the mixture of all the standards used. CA, GCA, TCA, CME, DHCA, DCA, CDCA, GDCA, GCDCA, UDCA, TDCA, TCDCA, LCA, TLCA, CHOL. 
For several years, a great variety of analytical methods for quantitative determination of bile acids in various biological materials have been described [19, 27, 73, 74], including HPLCUV assays [25, 38, 81, 83]. The different classes of bile acids have different absorption intensities to UV light, showing some limitations in their detection [27, 80]. The main disadvantages of these methods are the limited sensitivity and specificity of UV detection, especially in complex biological matrices, such as tissues and feces, due to the lack of a chromophore in the bile acid molecule [14, 66, 73, 84].

In our study, compounds showed greater absorbance at $200 \mathrm{~nm}$, demonstrated by their larger areas. As it was demonstrated before by other authors [27, 69]; free bile acids were harder to detect than conjugated ones. Tauro-conjugated bile acids showed greater absorbance values than their corresponding glyco-conjugated ones.

We could demonstrate not only the great resolution power of HPLC even with a UV detector, but also we achieved the resolution of the majority of the identified compounds in a relatively short time. In previous works, although they reported separation of different bile acid types, running times were longer [71].

The C-18 column was the most appropriated for the resolution of the majority of the compounds. Although when doing HPLC, there are several parameters that should be taken into account to achieve an efficient separation of all bile acids; among the most important ones, are the composition and strength of the mobile phase. The strength is involved in the peaks symmetry control and in the bile acid elution order [40, 93]; in this work, for most retained compounds we increased the mobile phase strength increasing the proportion of acetonitrile, so for example, we could elute cholesterol. Under these conditions, the analysis reproducibility in terms of retention time and areas between different runs, even among long periods of inactivity, was satisfactory, allowing a precise identification of the peaks. Moreover, the high efficiency of this chromatographic system was demonstrated and this is reflected in the peak sharpness.

As we found unidentified compounds which did not coincide with any of the standards used, we are in process of identifying them and also confirming the identity of fecal bile acids found by HPLC-UV, through ESI-MS/MS.

Finally, we were able to differentiate all Xenarthra species through their fecal bile acid patterns, by HPLC. This study is of great relevance because is the first one in reporting HPLC as an ecological tool for the identification of wild-collected mammal feces. Moreover, it has proved to be a relatively simple method, without large preparation and derivatization steps, achieving resolution and identification of most of the compounds in a short time.

\section{Conclusion}

As a non-invasive method, the analysis of feces is a fundamental tool for field work in ecological studies, not only to identify the presence of certain species in a particular area [94], but also for studying threatened species or animals difficult to observe and trap. For the identification, the original fecal shape must be maintained; however, as several factors can 
corrode it through time, visual identification is not always reliable. Particularly, feces from Xenarthra are sometimes difficult to identify in the wild because they are, commonly, total or partially mixed with the substrate.

The chromatographic determination of fecal bile acids has become a more precise method to identify unknown feces from the wild. The comparison of the whole pattern of fecal bile acids between field-collected scats and scats with known origin allows identifying the species from fecal material, avoiding capture and manipulation of animals.

We were able to establish the fecal bile acid patterns for Xenarthra species, which were different for all of them. Moreover, these patterns were consistent among different individuals of the same species. As it was reported before for other mammal species [3, 5-7, $25,35,36]$, we confirm that chromatographic determination of fecal bile acids is a precise technique to identify unknown wild-collected feces.

Chromatographic techniques are the method of choice for a detailed analysis of bile acid profiles in biological samples. However, there is no a single satisfactory method for the analysis of all bile acids in biological fluids. All techniques present limitations in their specificity, analysis times or simplicity. Some types of samples, such as urine or feces, can contain complex mixtures of bile acids; other samples, such as tissues and cells, can contain small quantities of bile acids, being, then, easier to analyze. Thus, the choice of the analytical method will depend on the particular aim of the study and the type of sample. Certainly, in our case, the use of multiple analytical techniques (TLC, HPLC, HPLC-ESI-MS/MS) allows a precise resolution and confirmation of complex bile acid patterns.

HPLC-UV analysis has been widely used for the determination of bile acids in several biological fluids [68]. The main disadvantage is its limited sensitivity and specificity to UV detection in complex biological samples, such as tissues and feces [66].

In our study, both techniques, TLC and HPLC, presented advantages and disadvantages in the analysis of Xenarthra feces. Although TLC offers advantages such as relative simplicity, short analysis times, ease of operation and simultaneous analysis of a big number of samples, as reported previously [43, 47, 93], it can be affected by external factors such as environmental conditions, humidity and temperature, and by the operability of the researcher. Moreover, it has lower resolution power and reproducibility than HPLC [44].

TLC separation selectivity allowed resolving and visualizing CHOL, free and amidated bile acids in a single run, as it was reported before [95]. TLC could also resolve pairs of bile acids with very similar Rf values, for example CA-GCA and DCA-CDCA. Previous works have reported the performance of both methodologies for bile acid analysis in gallbladder bile or liver. In [95] they reported that TLC produces quick, precise and reproducible results, with shorter analysis times and low costs.

On the other hand, HPLC most important advantages were precision, higher resolution power than TLC, high sensitivity and specificity, as it was observed by other authors before $[44,75]$. However, one disadvantage is longer analysis times due to the injection of only one sample at the time. 
Diet of animals, and particularly in the case of Xenarthra species, can have an effect on the detectability of some bile acids. In our study, in feces with high contents of vegetal material, pigments appeared as colored bands in the TLC chromatographic plates and unidentified peaks in HPLC. However, they did not interfere with bile acid detection and we were able to correctly identify compounds.

With the application of HPLC we corroborate TLC results, not only confirming the presence of the compounds found by TLC but also identifying new compounds which were not resolved by TLC, in Xenarthra feces.

Bile acid detection constituted an essential step in both techniques. The choice of the detection system is governed mainly by bile acid structures. In TLC, spraying the plates with the revealing reagent is the critical step, in which it is important the ability and experience of the operator. Spraying must be uniform to avoid uncolored areas that later interfere with the correct identification of spots. In HPLC, different classes of bile acids have different absorption capabilities to UV light; this is a critical factor especially for free bile acids, showing limitations for their detection. However, in our study we could detect free bile acids.

In relation to the components of the chromatographic system, the choice of the mobile and stationary phase is very important, as they depend on the type of sample and the objectives of the study. In TLC we used silica gel as stationary phase, which is the most used phase for lipid analysis [47]. The solvent system composed of toluene:acetic acid:water (5:5:1.5 v/v) proposed by [5] was the most adequate for our aims, achieving a good resolution of the great majority of the compounds, except for some tauro-conjugated bile acids such as TDCA and TLCA. In HPLC we used a reversed-phase column with a ODS stationary phase, and the mobile phase composed of ammonium carbonate and acetonitrile; under these conditions we were able to separate almost all bile acids, including tauro-conjugated bile acids not resolved by TLC.

Thus, our work demonstrates that both techniques, TLC and HPLC, are complementary, and they should be used together to take advantage of the positive aspects of each one.

The analysis of fecal bile acids is a useful tool not only for ecological and biological studies, but also is of great clinical interest. These determinations can be helpful in the evaluation of intestinal, biliary and hepatic functions, and in the diagnosis and treatment of some related diseases such as colon cancer $[46,96,97]$.

Chromatographic techniques have been widely used to identify bile acids in different biological materials, mainly in gallbladder bile of mammal species. However, our study is the first one to report the use of TLC and HPLC to differentiate Xenarthra species through their fecal bile acid patterns.

Finally, our work established the validity of fecal bile acids to differentiate close related species, being useful to assess habitat use and to study food habits of sympatric species. The determination of these species-specific patterns offers robust data for the elaboration of conservation strategies in the long term. 


\section{Author details}

Emma B. Casanave

Cátedra de Fisiología Animal, Dpto. de Biología, Bioquímica y Farmacia,

Universidad Nacional del Sur, Bahía Blanca, Buenos Aires, Argentina

Consejo Nacional de Investigaciones Científicas y Técnicas, (CONICET) Argentina

M. Soledad Araujo

Cátedra de Fisiología Animal, Dpto. de Biología, Bioquímica y Farmacia,

Universidad Nacional del Sur, Bahía Blanca, Buenos Aires, Argentina

Gustavo H. López

Cátedra de Bioanalítica II, Dpto. de Biología, Bioquímica y Farmacia,

Universidad Nacional del Sur, Bahía Blanca, Buenos Aires, Argentina

\section{Abbreviation list}

CA: Cholic acid

CDCA: Chenodeoxycholic acid

CHOL: Cholesterol

CME: Cholic-methyl-esther

DCA: Deoxycholic acid

DHCA: Dehydrocholic acid

EIA: Enzyme immunoassays

ELSD: Evaporative Light Scattering Detector

ESI: Electrospray Ionization

FAB: Fast Atom Bombardment

FLD: Fluorescence Detector

GC: Gas Chromatography

GCA: Glycocholic acid

GCDCA: Glycochenodeoxycholic acid

GDCA: Glycodeoxycholic acid

HPLC: High Performance Liquid Chromatography

HPTLC: High Performance Thin Layer Chromatography

LC: Liquid Chromatography

LCA: Lithocholic acid

MS: Mass Spectrometry

ODS: octadecylsilane

RIA: Radioimmunoanalysis

RID: Refractive Index Detector

TCA: Taurocholic acid

TCDCA: Taurochenodeoxycholic acid

TDCA: Taurodeoxycholic acid

TLC: Thin layer Chromatography 
TLCA: Taurolithocholic acid

UDCA: Ursodeoxycholicacid

UV: Ultraviolet

\section{Acknowledgement}

This work was supported by Secretaría General de Ciencia y Tecnología, Universidad Nacional del Sur, PGI 24/B152.

\section{References}

[1] Soler L.; Lucherini, M.; Manfredi, M.C.; Ciuccio, M.; Birochio D. \& Casanave E. B. (2009). Characteristics of defecation sites in the Geoffroy's Cat Leopardus geoffroyi. Mastozoología Neotropical. 16(2): 485-489.

[2] Castillo, D.F.; Birochio D.E.; Lucherini, M. \& Casanave, E.B. (2011). Diet of adults and cubs of Lycalopexgymnocercusin Pampas grassland: a validation of the Optimal Foraging Theory?. Annales Zoologici Fennici 48: 251-256.

[3] Capurro, A.F., Novaro, A.J.; Travaini, A. \& Romero, M. (1997). Improved bile acid thin layer chromatography to identify feces of Neotropical carnivores. Journal of Wildlife Management 61: 1424-1427.

[4] Chame, M. (2003). Terrestrial Mammal Feces: a Morphometric Summary and description. Memórias do Instituto Oswaldo Cruz 98: 71-94.

[5] Cazón, A.V. \& Sühring, S.S. (1999). A technique for extraction and thin layer chromatography visualization of fecal bile acids applied to Neotropical felid scats. Revista de Biología Tropical 47: 245-249.

[6] Guerrero, C.; Espinoza, L.;Niemeyer, H.M. \& Simonetti, J.A. (2006). Empleo del perfil de ácidos biliares fecales para evaluar uso del hábitat por carnívoros amenazados en el bosque maulino de Chile central. Revista Chilena de Historia Natural 79: 89-95.

[7] Khorozyan, I.G.; Cazón, A.V.; Malkhasyan, A.G. \& Abramov, A.V. (2007). Using ThinLayer Chromatography of Fecal Bile Acids to Study the Leopard (Panthera parduscis caucasica) Population.Biology Bulletin 34: 361-366.

[8] Araujo, M.S.; Ciuccio, M.; Cazón, A.V. \& Casanave, E.B. (2010). Differentiation of Xenarthra (Mammalia) species through the identification of their fecal bile acid patterns: an ecological tool. 2010. Revista Chilena de Historia Natural 83: 557-566.

[9] Hofmann, A.F.; Hagey, L.R.; Krasowski, M.D. (2010). Bile salts of vertebrates: structural variation and possible evolutionary significance. Journal of Lipid Research 51: 226-246.

[10] Hagey, L.R.; Vidal, N.; Hofmann, A.F. \& Krasowski, M.D. (2010a) Evolutionary diversity of bile salts in reptiles and mammals, including analysis of ancient human and extinct giant ground sloth coprolites. BMC Evolutionary Biology 10: 133.

[11] Hofmann, A.F. \& Hagey, L.R. (2008). Bile Acids: Chemistry, Pathochemistry, Biology, Pathobiology and Therapautics. Cellular and Molecular Life Sciences 65: 2461-2483.

[12] Street, J.M., Trafford, D.J.H. \& Makin, H.L.J. (1983). The quantitative estimation of bile acids and their conjugates in human biological fluids. Journal of Lipid Research 24: 491511. 
[13] Russell, D.W. (2003). The enzymes, regulation, and genetics of bile acid synthesis. Annual Reviews in Biochemistry 72: 137-174.

[14] Alnouti, Y.; Csanaky, I.L. \& Klaassen, C.D. (2008). Quantitative-profiling of bile acids and their conjugates in mouse liver, bile, plasma, and urine using LC-MS/MS. Journal of Chromatography B 873: 209-217.

[15] Mukhopadhyay, S. \& Maitra, U. (2004). Chemistry and biology of bile acids. Current Science 87 (12): 1666-1683.

[16] Eneroth, P.B.; Gordon, B.;Ryhage, R.R. \& Sjövall, J. (1966a). Identification of mono and dihydroxy bile acids in human feces by gas-liquid chromatography and mass spectrometry. Journal of Lipid Research 7: 511-523.

[17] Eneroth, P.B.; Gordon, R. \& Sjövall, J. (1966b). Characterization of trisubstituted cholanoic acids in human feces. Journal of Lipid Research 7: 524-530.

[18] Sjövall, J. (2004). Fifty years with bile acids and steroids in health and disease. Lipids 39(8): 703-722.

[19] Batta, A.K. \& Salen, G. (1999). Gas chromatography of bile acids. Journal of Chromatography B. Biomedical Sciences and Applications 723(1-2): 1-16.

[20] [20] Huang, J.; Bathena, S.P.R.;Csanaky, I.L. \& Alnouti, Y. (2011). Simultaneous characterization of bile acids and their sulfate metabolites in in mouse liver, plasma, bile, and urine using LC-MS/MS. Journal of Pharmaceutical and Biomedical Analysis.Journal of Pharmaceutical and Biomedical Analysis 55 (5): 1111-1119.

[21] Eneroth, P. (1963). Thin-layer chromatography of bile acids. Journal of Lipid Research 4(1): 11-16.

[22] Zarzycki, P.K.; Wierzbowska, M. \& Lamparczyk, H. (1999). Retention and separation studies of cholesterol and bile acids using thermostated thin-layer chromatography. Journal of Chromatography A 857: 255-262.

[23] Grundy, S.M.; Ahrens, E.H. \& Miettinen, T.A. (1965). Quantitative isolation and gasliquid chromatographic analysis of total fecal bile acids.Journal of Lipid Research 6: 397410.

[24] Batta, A.K.;Salen, G.; Batta, P.; Tint, G.S.;Alberts, D. \& Earnest, D.L. (2002). Simultaneous quantitation of fatty acids, sterols and bile acids in human stool by capillary gas-liquid chromatography. Journal of Chromatography B 775: 153-161.

[25] Yeh, Y.H. \& Hwang, D.F. (2001). High-Performance Liquid Chromatographic determination for bile components in fish, chicken and duck. Journal of Chromatography B 751: 1-8.

[26] Zhang, C.; Brown, S.B. \& Hara, T.J. (2001). Biochemical and physiological evidence that bile acids produced and released by lake char (Salvelinusnamaycush) function as chemical signals. Journal of Comparative Physiology B 171: 161-171.

[27] Kakiyama, G.; Hosoda, A.; Iida, T.; Fujimoto, Y.; Goto, T.; Mano, T.; Goto, J. \& Nambara, T. (2006). A direct method for the separation and quantification of bile acid acyl glycosides by High-Performance Liquid Chromatography with an Evaporative Light Scattering Detector. Journal of Chromatography A 1125: 112-116.

[28] Beckett, G.J.; Hunter, W.M. \& Percy-Robb, I.W. (1978). Investigations into the choice of immunogen, ligand, antiserum and assay conditions for the radioimmunoassay of conjugated cholic acid.ClinicaChimicaActa 88 (2): 257-266. 
[29] Simmonds, W.J.; Korman, M.G.; Go, V.L. \&Hofmann, A.F. (1973). Radioimmunoassay of conjugated cholyl bile acids in serum. Gastroenterology 65(5): 705-11.

[30] Demers L.M. \&Hepner G. (1976). Radioimmunoassay of bile acids in serum. Clinical Chemistry 22(5): 602-606.

[31] Baqir, Y.A.;Murison, J.; Ross, P.E. \& Bouchier, I.A. (1979). Radioimmunoassay of primary bile salts in serum. Journal of Clinical Pathology 32:560-564. doi:10.1136/jcp.32.6.560.

[32] Hagey, L.R.; Crombie, D.L.; Espinosa, E.; Carey, M.C.; Igimi, H. \& Hofmann, A.F. (1993). Ursodeoxycholic acid in the Ursidae: biliary bile acids of bears, pandas, and related carnivores. Journal of Lipid Research 34: 1911-1917.

[33] Picton, H.D. \& Kendall, K.C. (1994). Chromatographic (TLC) differentiation of grizzly bear and black bear scats. International Conference Bear Research and Management 9: 497-501.

[34] Jiménez, J.E.;Yañez, J.L. \& Jaksic, F.M. (1996). Inability of Thin layer chromatography to distinguish feces from congeneric foxes by their bile acid contents.Acta Theriologica 41: 211-215.

[35] Fernandez, G.J.;Corley, J.C. \& Capurro, A.F. (1997). Identification of cougar and jaguar feces through bile acid chromatography. Journal of Wildlife Management 61: 506-510.

[36] Taber, A.B.; Novaro, A.J.; Neris, N. \& Colman, F.H. (1997). The food habits of sympatric jaguar and puma in the Paraguayan Chaco. Biotropica 29: 204-213.

[37] Solá, S.; Garshelis, D.L.; Amaral, J.D.; Noyce, K.V.; Coy, P.L.; Steer, C.J.; Iaizzo, P.A. \& Rodrigues, C.M.P. (2006). Plasma levels of ursodeoxycholic acid in black bears, Ursusamericanus: seasonal changes. Comparative Biochemistry and Physiology, Part C 143: 204-208.

[38] Kuroki, S.; Schteingart, C.D.; Hagey, L.R.; Cohen, B.I.; Mosbach, E.H.; Rossi, S.S.; Hofmann, A.F.; Matoba, M.; Une, M. \& Hoshita, T. (1988). Bile salts of the West Indian manatee, Trichechusmanatus latirostris: novel bile alcohol sulfates and absence of bile acids. Journal of Lipid Research 29: 509-522.

[39] Hagey, L.R.; Schteingart, C.D.; Ton-Nu, H.T. \& Hofmann, A.F. (2002). A novel primary bile acid in the Shoebill stork and herons and its phylogenetic significance.Journal of Lipid Research 43: 685-690.

[40] Scalia, S. (1995). Bile acid separation. Journal of Chromatography B 671: 299-317.

[41] Pyka, A. \&Dolowy, M. (2004). Separation of selected bile acids by TLC. III. Separation on Various Stationary Phases. Journal of Liquid Chromatography \& Related Technologies 27(16): 2613-2623.

[42] Pyka, A. \& Dolowy, M. (2005). Separation of selected bile acids by TLC. VII. Separation by reversed partition HPTLC. Journal of Liquid Chromatography \& Related Technologies 28: 1573-1581.

[43] Sherma, J. \& Fried, B. (2005). Thin layer chromatographic analysis of biological samples. A review. Journal of Liquid Chromatography \& Related Technologies 28: 2297-2314.

[44] Peterson, B.L. \& Cummings, B.S. (2006). A review of chromatographic methods for the assessment of phospholipids in biological samples. Biomedical Chromatography 20: 227-243. 
[45] Dolowy, M. (2007). Separation of selected bile acids by TLC. IX. Separation on Silica gel 60 and on silica gel 60F254 aluminum plates impregnated with $\mathrm{Cu}$ (II), Ni (II), Fe (II) and Mn (II) cations. Journal of Liquid Chromatography and Related Technologies 30: 405-418.

[46] Perwaiz, S.; Tuchweber, B.; Mignault, D.; Gilat, T. \& Yousef, I.M. (2001). Determination of bile acids in biological fluids by liquid chromatography-electrospray tandem mass spectrometry. Journal of Lipid Research 42: 114-119.

[47] Palamarev, C.E.; Kandinska, M.I. \& Palamareva, M.D. (2004). Automatic Selection of Mobile Phases. IV. Thin-Layer Chromatography on Silica of Simple and Complex Organic Compounds. Journal of Liquid Chromatography \& Related Technologies 27: 2003-2018.

[48] Major, M.; Johnson, M.K.; Davis, W.S. \&Kellog, T.F. (1980). Identifying scats by recovery of bile acids. Journal of Wildlife Management 44: 290-293.

[49] Johnson, M.K.; Belden, R. \&Aldred, D.R. (1984). Differentiating mountain lion and bobcat scats. Journal of Wildlife Management 48: 239-244.

[50] Quinn, T. \& Jackman, W.R. (1994). Influence of diet on detection of fecal bile acids by thin-layer chromatography. Journal of Wildlife Management 58: 295-299.

[51] Redford, K.H. \& Wetzel, R.M. (1985). “Euphractus sexcinctus”. Mammalian Species 252: $1-4$.

[52] Casanave, E.B.; Manfredi, C. \& Luengos Vidal, E.M. (2003). Ecología comportamental de los armadillos en un pastizal serrano, In: Actas de las II Jornadas Interdisciplinarias del Sudoeste Bonaerense, María del C. Vaquero y Mabel N. Cernadas de Bulnes (eds), pp. 115-125. EdiUNS, ISBN 987-9281-76-4, Bahía Blanca, Argentina.

[53] Dalponte, J.C. \& Tavares-Filho, J.A. (2004). Diet of the yellow armadillo, Euphractus sexcinctus, in south-central Brazil. Edentata 6: 37-41.

[54] Soibelzon, E.; Daniele, G.; Negrete, J.; Carlini, A.A. \& Plischuk, S. (2007). Annual diet of the little hairy armadillo, Chaetophractusvellerosus (Mammalia, Dasypodidae) in Buenos Aires Province, Argentina. Journal of Mammalogy 88: 1319-1324.

[55] McDonough, C.M. \& Loughry, W.J. (2008). Behavioral ecology of armadillos, in: Vizcaíno, S.F. \& W.J. Loughry (eds.), The biology of the Xenarthra, Univ. Press of Florida, Gainesville, pp. 281-293.

[56] Ray, J.C. (1996). Resource use patterns among mongooses and other carnivores in a central African rainforest. Ph.D. dissertation, University of Florida, Gainesville.

[57] Ray, J.C. \& Sunquist, M.E. (2001). Trophic relations in a community of African rainforest carnivores. Oecologia 127: 395-408.

[58] Hofmann, A.F. (1961). Thin-layer adsorption chromatography of free and conjugated bile acids on silicic acid. Journal of Lipid Research Notes on Methodology 3: 127-128.

[59] Chavez, M.N. \& Krone, C.L. (1976). Silicic acid Thin-Layer Chromatography of conjugated and free bile acids. Journal of Lipid Research 17: 545-547.

[60] Kritchevsky, D.;Martak, D.S. \& Rothblat, G.H. (1963). Detection of bile acids in thinlayer chromatography. Analytical Biochemistry 5(5): 388-392.

[61] Sarbu, C.; Kuhajda, K. \& Kevresan, S. (2001). Evaluation of the lipophilicity of bile acids and their derivatives by thin-layer chromatography and principal component analysis. Journal of Chromatography A 917: 361-366. 
[62] Setchell, K.D.R.; Schwarz, M.; O'Connell, N.C.; Lund, E.G.; Davis, D.L.; Lathe, R.; Thompson, H.R.; Tyson, W.R.; Sokol, R.J. \& Russell, D.W. (1998). Identification of a New Inborn Error in Bile Acid Synthesis: Mutation of the Oxysterol $7 \alpha$-Hydroxylase Gene Causes Severe Neonatal Liver Disease. Journal of Clinical Investigations 102: 1690-1703.

[63] Keller, S. \& Jahreis, G. (2004). Determination of underivatised sterols and bile acid trimethylsilyl ether methyl esters by gas chromatography-mass spectrometry-single ion monitoring in faeces. Journal of Chromatography B 813: 199-207.

[64] Hagey, L.R.; Vidal, N.; Hofmann, A.F. \& Krasowski, M.D. (2010b). Complex evolution of bile salts in birds. Auk. 127(4): 820-831. doi:10.1525/auk.2010.09155.

[65] Hagey, L.R.; Møller, P.R.; Hofmann, A.F. \& Krasowski, M.D. (2010c). Diversity of bile salts in fish and amphibians: evolution of a complex biochemical pathway. Physiological and Biochemical Zoology 83: 308-321.

[66] Roda, A.; Piazza, F. \& Baraldini, M. (1998). Separation techniques for bile salts analysis. Journal of Chromatography B 717: 263-278.

[67] Setchell, K.D.; Lawson, A.M.; Tanida, N. \& Sjövall, J. (1983). General methods for the analysis of metabolic profiles of bile acids and related compounds in feces. Journal of Lipid Research 24: 1085-1100.

[68] Eneroth, P., Hellstrom, K. \& Sjövall, J. (1968). A method for quantitative determination of bile acids in human feces. Acta Chemica Scandinavica 22: 1729-1744.

[69] Shaw, R. \& Elliott, W.H. (1978). Bile acids. LVII. Analysis of bile acids by high-pressure liquid chromatography and mass spectrometry. Lipids 13: 971-975.

[70] Rossi, S.S.; Converse, J.L. \& Hofmann, A.F. (1987). High pressure liquid chromatography analysis of conjugated bile acids in human bile: simultaneous resolution of sulfated and unsulfated lithocholyl amidates and the common conjugated bile acids. Journal of Lipid Research 28: 589-595.

[71] Prydz, K.; Kase, B.F. \& Pedersen, J.I. (1988). High pressure liquid chromatography solvent systems for studies of bile acid biosynthesis. Journal of Lipid Research 29: 532537.

[72] Sakakura, H.; Kimura N.; Takeda, H.; Komatsu, H.; Ishizaki, K. \& Nagata, S. (1998). Simultaneous determination of bile acids in rat liver tissue by high-performance liquid chromatography. Journal of Chromatography B 178: 33-40.

[73] Lin, D-L.; Chang, H-C. \& Chen, C-Y. (2000). Identification and quantitation of bile acids in bear bile by HPLC. Journal of Food and Drug Analysis 8(4): 283-288.

[74] Burkard, I.; Von eckardstein, A. \& Rentsch, K.M. (2005). Differentiated quantification of human bile acids in serum by high-performance liquid chromatography-tandem mass spectrometry. Journal of Chromatography B 826: 147-159.

[75] Persson, E.; Lofgren, L.; Hansson, G.; Abrahamsson, B.; Lennernas, H. \& Nilsson, R. (2007). Simultaneous assessment of lipid classes and bile acids in human intestinal fluid by solid-phase extraction and HPLC methods. Journal of Lipid Research 48: 242-251.

[76] Lima, E.S. \& Abadía, D.S.P. (2002). High-Performance Liquid Chromatography of fatty acids in biological samples. Analytica Chimica Acta 465(1-2): 81-91.

[77] Robards, K.; Haddad, P.R. \& Jackson, P.E. (1994). Principles and Practice of Modern Chromatographic Methods, Academic Press, London, San Diego, pp. 495. 
[78] Ardrey, R.E. (2003). Liquid Chromatography, in: John Wiley \& Sons, Ltd. (eds.), Liquid Chromatography-Mass Spectrometry: An introduction. The Atrium, Southern Gate, Chichester, West Sussex, England, pp. 7-31.

[79] Parris, N.A. (1977). Liquid chromatography separation of bile acids. Journal of Chromatography 133(2): 273-279.

[80] Sjövall, J.; Griffiths, W.J.; Setchell, K.D.R.; Mano, N. \& Goto, J. (2010), Analysis of Bile Acids, in: Makin, H.L.J. \& Gower, D.B. (eds.). Steroid Analysis, $2^{\text {nd }}$ edition, Springer, pp. 837-966.

[81] [80] Torchia, E.C.;Labonté, E.D. \& Angellon, L.B. (2001). Separation and quantitation of bile acids using an isocratic solvent system for High Performance Liquid Chromatography coupled to an Evaporative Light Scattering Detector. Analytical Biochemistry 298: 293-298.

[82] Carducci, C.N.; Matejka, M.; Ergüven, H.; Labonia, N. \& Mamianetti, A. (1985). Highperformance liquid chromatographic analysis of bile acids in hamster bile. Journal of Chromatography 337(1): 91-97.

[83] Mamianetti, A.; Garrido, D.; Carducci, C.N. \& Vescina, M.C. (1999). Fecal bileacidexcretionprofile in gallstonepatients. Medicina 59 (3): 269-273.

[84] Natalini, B.; Sardella, R.; Camaioni, E.; Natalini, S. \& Pellicciari, R. (2006). Dominant factors affecting the chromatographic behaviour of bile acids. Chromatographia 64(5): 343-349.

[85] Griffiths, W.J. \& Sjövall, J. (2010). Bile acids: analysis in biological fluids and tissues. Journal of Lipid Research 51: 23-41.

[86] Kamada, S.M.; Maeda, M. \& Tsuji, A. (1983). Fluorescence high-performance liquid chromatographic determination of free and conjugated bile acids in serum and bile using 1-bromoacetylpyrene as a pre-labeling reagent. Journal of Chromatography 272: 29-41.

[87] Roda A.; Gioacchini, A.M.; Cerrè, C. \& Baraldini, M. (1995). High-performance liquid chromatographic-electrospray mass spectrometric analysis of bile acids in biological fluids.Journal of Chromatography B: Biomedical Sciences and Applications 665 (2): 281294.

[88] Bootsma, A.H.; Overmars, H.; Van rooij, A.; Van lint, A.E.M.; Wanders, R.J.A.; Van Gennip, A.H. \& Vreken, P. (1999). Rapid analysis of conjugated bile acids in plasma using electrospray tandem mass spectrometry: Application for selective screening of peroxisomal disorders. Journal of Inherited Metabolic Disease 22 (3): 307-310.

[89] Hong, Y.J.; Turowski, M.; Lin, J.T. \& Yokoyama, W.H. (2007). Simultaneous characterization of bile acid, sterols, and determination of acylglycerides in feces from soluble cellulose-fed hamsters using HPLC with Evaporative Light-Scattering Detection and APCI-MS. Journal of Agricultural and Food Chemistry 55(24): 9750-9757.

[90] Griffiths, W.J.; Jonsson, A.P.; Liu, S.; Rai, D.K. \& Wang, Y. (2001). Electrospray and tandem mass spectrometry in biochemistry. Biochemestry Journal 355: 545-561.

[91] Tomer, K.B.; Jensen, N.J.; Gross, M.L. \& Whitney, J. (1986). Fast atom bombardment with tandem mass spectrometry for determination of bile salts and their conjugates. Biomedical and Environmental Mass Spectrometry 13: 265-272. 
[92] Chatman, K.; Hollenbeck, T.; Hagey, L.; Vallee, M.; Purdy, R.; Weiss, F. \& Siuzdak, G. (1999). Nanoelectrospray Mass Spectrometry and Precursor Ion Monitoring for Quantitative Steroid Analysis and Attomole Sensitivity. Analytical Chemistry 71 (13): 2358-2363.

[93] Zhang, Y.K.J.; Guo, G.L. \& Klaassen, C.D. (2011). Diurnal Variations of Mouse Plasma and Hepatic Bile Acid Concentrations as well as Expression of Biosynthetic Enzymes and Transporters. PLoS ONE 6(2): e16683. doi:10.1371/journal.pone.0016683.

[94] Zarzycki, P.K.; Kulhanek, K.M.; Smith, R.; Bartoszuk, M.A. \& Lamparczyk, H. (2005). Planar Chromatography versus Column Chromatography: A Performance Comparison. LCGC North America 23(3): 286-300.

[95] Ciuccio, M.; Araujo, M.S. \& Casanave, E.B. (2007). Estudio ecológico de las especies de armadillos presentes en cercanías de Bahía Blanca. In Ambiente natural, campo y ciudad: Estrategias de uso y conservación en el Sudoeste Bonaerense. N. Cazzaniga y María del C. Vaquero, pp. 183-190, EdiUNS, ISBN 978-987-23429-1-3, Bahía Blanca, Argentina.

[96] Müllner, S., Hofmann, R., Saar, K. \& Karbe-Thönges, B. (1992). Economic assay for the evaluation of bile acid sequestrants using ox bile and quantitative TLC analysis.Journal of planar chromatography, modern TLC 5(6): 408-416.

[97] Kasbo, J.; Saleem, M.; Perwaiz, S.; Mignault, D.; Lamireau, T.; Tuchweber, B. \& Yousef, I. (2002). Biliary, fecal and plasma deoxycholic acid in rabbit, hamster, guinea pig, and rat: Comparative study and implication in colon cancer. Biological and Pharmaceutical Bulletin 25(10): 1381-1384.

[98] Meance, S.; Boutron-Ruault, M.C.; Myara, A.; Gerhardt, M.F.; Marteau, P.; Lavergne, A.; Franchisseur, C. \& Bouley, C. (2003). Fecal primary bile acids and serum cholesterol are associated with colorectal adenomas. Digestive Diseases and Sciences 48(9): 1751-1757. 